Check for updates

Cite this: RSC Adv., 2018, 8, 12635

Received 8th February 2018

Accepted 26th March 2018

DOI: $10.1039 / c 8 r a 01236 a$

rsc.li/rsc-advances

\title{
Divergent synthesis of dual 1,4-dihydropyridines with different substituted patterns from enaminones and aldehydes through domino reactions $\uparrow$
}

\author{
Fu-Jun Liu, ${ }^{a}$ Tian-Tian Sun, ${ }^{a}$ Yun-Gang Yang, ${ }^{a}$ Chao Huang ${ }^{\star b}$ \\ and Xue-Bing Chen iD *a
}

\begin{abstract}
A concise and efficient protocol for the regioselective synthesis of dual 1,4-dihydropyridines with several substituted patterns has been developed from a cascade cyclization of enaminones and aldehydes in different media $\left(\mathrm{EtOH} / \mathrm{CH}_{3} \mathrm{CN}\right.$ ). The one-pot cascade reaction involves at least five reactive sites and generates multiple $\mathrm{C}-\mathrm{C}$ and $\mathrm{C}-\mathrm{N}$ bonds. The established protocol explores the chemistry of enaminones by employing their three reactive sites. The method has several advantages including mild conditions, operational simplicity, and high bond-forming efficiency. It may offer promise in a variety of biochemical applications.
\end{abstract}

\section{Introduction}

Molecularly diverse and complex heterocycles play an important role in both organic synthesis and discovery of new pharmaceutical reagents. ${ }^{1}$ 1,4-Dihydropyridines (1,4-DHPs) derivatives represent one of the most important classes of heterocycles due to their application in biological activities and molecular functions. For example, 1,4-DHPs are contained in marketed drugs such as the calcium channel blockers nifedipine, felodipine, nicardipine, and nimodipine. During the last several decades, it has been demonstrated that 1,4-DHPs are involved in various biological applications, i.e. antibacterial, ${ }^{2}$ anticonvulsant, ${ }^{3}$ antiHIV ${ }^{4}$ anti-tumour, ${ }^{5}$ radioprotective, ${ }^{6}$ and neuroprotection ${ }^{7}$ activities; they could also act as adenosine-A3 receptor antagonism ${ }^{8}$ and sirtuin inhibitor. ${ }^{9}$ In view of their high significance, numerous methodologies for 1,4-DHPs have been introduced. ${ }^{10}$ However, few strategies have been successfully developed for efficient and concise access to highly functionalized and molecularly diverse target products. Therefore, it is of great importance to build libraries of target compounds in a parallel manner.

Multicomponent domino reactions (MCDRs) are powerful tools in modern organic synthesis because they enable more than

${ }^{a}$ Key Laboratory of Natural Pharmaceutical and Chemical Biology of Yunnan Province, School of Science, Honghe University, Mengzi, Yunnan 661100, China. E-mail: orangekaka@126.com

${ }^{b}$ School of Chemistry and Environment, Engineering Research Center of Biopolymer Functional Materials of Yunnan, Yunnan Minzu University, Kunming, Yunnan 650503, China

$\dagger$ Electronic supplementary information (ESI) available: Detailed experimental procedures. CCDC 1819954. For ESI and crystallographic data in CIF or other electronic format see DOI: 10.1039/c8ra01236a two building blocks to combine in a single-step operation to generate complex structures through the simultaneous formation of multiple bonds. ${ }^{11}$ With their inherent characteristics, i.e., step efficiency, atom economy, target molecules diversity, operational simplicity. MCDRs have become an essential tool for generating complex molecular libraries in the screening of biologically and pharmacologically active candidates.

As a group of versatile and powerful synthons, enaminones have been used for construction of diverse heterocyclic systems. ${ }^{12}$ These compounds include acridines, ${ }^{13}$ indoles, ${ }^{14}$ naphthyridines,${ }^{15}$ quinolones, ${ }^{16}$ pyrroles, ${ }^{17}$ thiazines, ${ }^{18}$ pyridines, ${ }^{19}$ and thiazoles. ${ }^{20}$ Some of these heterocycles which are synthesized from enaminones also demonstrate promising biological activity. ${ }^{21}$ So far, a number of natural product-like heterocycles have been successfully synthesized based on enaminones. Their synthetic methodology and associated medicinal activity should be engaged in the chemistry community.

In pursuit of our research interests regarding the development of MCRs based on enaminones, we report a convenient and efficient protocol for the regioselective synthesis of two libraries of 1,4-DHPs 3 and $\mathbf{4}$ via a tandem annulation of enaminones 1 and aldehydes 2 in EtOH and $\mathrm{CH}_{3} \mathrm{CN}$, respectively (Scheme 1). At least five distinct reactive sites participate in this process.

\section{Results and discussion}

On the basis of our recent work, we examined the domino reaction which employed $N-p$-F-Ph substituted enaminone 1a and 4-chlorobenzaldehyde $\mathbf{2 a}$ as the substrates (Table 1 ). 


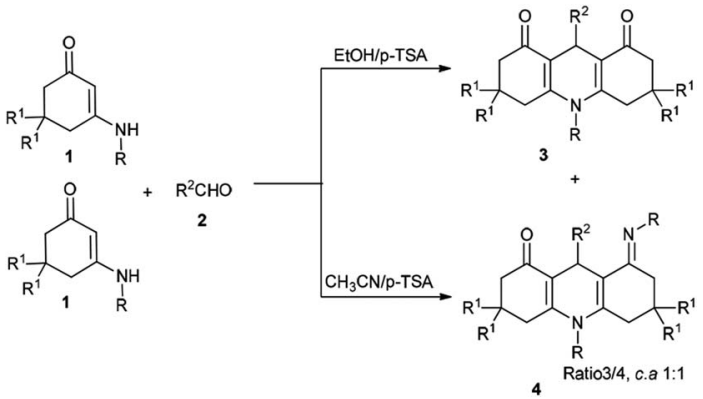

Scheme 1 Proposed mechanism for the construction of 1,4-DHPs 3 and 4 .

First, the reaction was executed without any catalyst in anhydrous ethanol at reflux; no reaction occurred even within 3 hours (Table 1, entry 1). Next, different types of organic bases, such as piperidine, $\mathrm{Et}_{3} \mathrm{~N}$, DBU, and $\mathrm{DABCO}$, were employed as catalysts, but no transformation was observed (Table 1, entries 2-5). Nevertheless, when $p$-TSA (0.2 equiv.) was added, compound 3a was obtained in a yield of $66 \%$ (Table 1 , entry 6 ). Other acids, such as TFA, HOAc and L-proline were also investigated, but could not generate the reaction efficiently (Table 1, entries 7-9). The reaction conditions were then optimized by screening several solvents. When $p$-TSA was added to a solution of 1a and 2a in $\mathrm{CH}_{3} \mathrm{CN}$ (Table 1, entry 10), a new compound $4 \mathbf{a}$

Table 1 Optimization of the reaction conditions for the model reaction $^{a}$

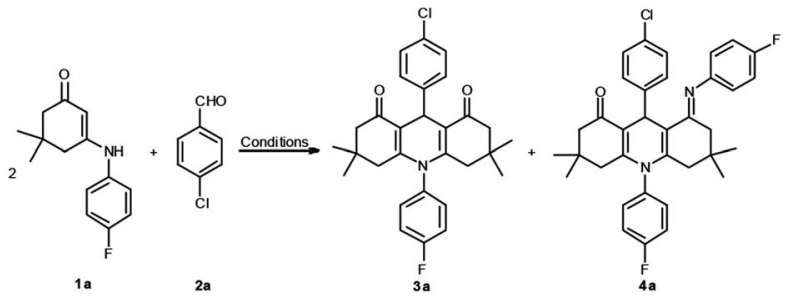

\begin{tabular}{|c|c|c|c|c|c|}
\hline \multirow[b]{2}{*}{ Entry } & \multirow[b]{2}{*}{ Solvent } & \multirow[b]{2}{*}{ Catalyst $^{b}$} & \multirow[b]{2}{*}{ Time (h) } & \multicolumn{2}{|c|}{ Yield $^{c}(\%)$} \\
\hline & & & & $3 a$ & $4 a$ \\
\hline 1 & EtOH & - & 4 & n.r & n.r \\
\hline 2 & EtOH & Piperidine & 3 & n.r & n.r \\
\hline 3 & EtOH & $\mathrm{Et}_{3} \mathrm{~N}$ & 3 & n.r & n.r \\
\hline 4 & EtOH & DBU & 3 & n.r & n.r \\
\hline 5 & EtOH & DABCO & 3 & n.r & n.r \\
\hline 6 & EtOH & $p$-TSA & 3 & 66 & n.r \\
\hline 7 & EtOH & TFA & 2 & 64 & n.r \\
\hline 8 & EtOH & HOAc & 2 & n.r & n.r \\
\hline 9 & EtOH & L-proline & 2 & n.r & n.r \\
\hline 10 & $\mathrm{CH}_{3} \mathrm{CN}$ & $p$-TSA & 2 & 43 & 36 \\
\hline 11 & $\mathrm{CH}_{3} \mathrm{CN}$ & TFA & 2 & 50 & 34 \\
\hline 12 & $\mathrm{CH}_{3} \mathrm{CN}$ & TfOH & 2 & n.r & Trace \\
\hline 13 & 1,4-Dioxane & $p$-TSA & 2 & 30 & 25 \\
\hline 14 & DMF & $p$-TSA & 2 & 45 & 28 \\
\hline 15 & $\mathrm{CH}_{3} \mathrm{OH}$ & $p$-TSA & 2 & n.r & n.r \\
\hline
\end{tabular}

${ }^{a}$ The reaction was performed with $1 \mathrm{a}(1.0 \mathrm{mmol}), 2 \mathrm{a}(0.6 \mathrm{mmol})$, and the solvent $(15 \mathrm{~mL})$ was under reflux temperature. ${ }^{b}$ Catalyst $(0.3$ $\mathrm{mmol}$ ) was added to the reaction. ${ }^{c}$ Isolated yields were based on enaminone 1a. was generated in $36 \%$ yield. In order to efficiently obtain a single final product, additional attempts were made. For example, three types of solvents, i.e., 1,4-dioxane, DMF, and $\mathrm{CH}_{3} \mathrm{OH}$, and acidic catalysts TFA, and TfOH were used in the reaction. However, the yields of $3 \mathbf{a}$ and $\mathbf{4 a}$ were decreased, and likewise, the vying preference for the formation of $\mathbf{4 a}$ was unsatisfied (Table 1, entries 11-15). Thus, ethanol was the best medium for selectively obtaining product 3a. It was discovered that the optimum reaction conditions for the synthesis of $\mathbf{3 a}$ include $p$-TSA as the catalyst and $\mathrm{EtOH}$ as the medium at reflux for $3 \mathrm{~h}$. Conversely, the best conditions for the preparation of $\mathbf{4 a}$ are conducted in $\mathrm{CH}_{3} \mathrm{CN}$.

With the optimal reaction conditions, the synthesis of 1,4DHPs 4 was tested by using a range of readily available enaminones and different substituted aldehydes. As shown in Table 2, for precursors 1 and 2 bearing either electron-withdrawing or electron-donating substituents on the aromatic ring, or the aromatic groups (for 2) with diverse substitution patterns (para or meta), all reactions proceeded smoothly to provide the corresponding 1,4-DHPs derivatives. The reaction was also investigated by using alkyl enaminone $\mathbf{1 h}$, which yielded the corresponding target molecule $\mathbf{3 m}$.

After completing the synthesis of compounds 3, the domino reaction was studied for preparing other target molecules 4 (Scheme 2). First, the scope of substrates 1 was tested by using 3-amino-5,5-dimethylcyclohex-2-enones. The reaction results of various 3-aminocyclohex-2-enonephenyls with aldehydes

Table 2 Substrate scope of 1 and synthesis of target molecules $3^{a}$
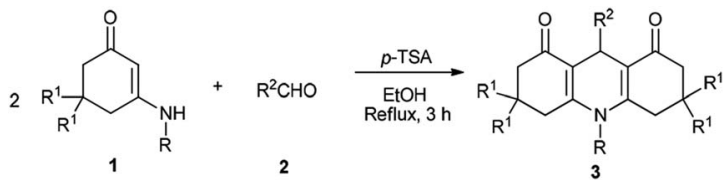

\begin{tabular}{|c|c|c|c|c|}
\hline Entry & $1\left(\mathrm{R} / \mathrm{R}^{1}\right)$ & $2\left(\mathrm{R}^{2}\right)$ & 3 & Yield $^{b}(\%)$ \\
\hline 1 & $1 \mathbf{a}\left(4-\mathrm{FC}_{6} \mathrm{H}_{5} / \mathrm{CH}_{3}\right)$ & $2 \mathbf{a}\left(4-\mathrm{ClC}_{6} \mathrm{H}_{5}\right)$ & $3 a$ & 66 \\
\hline 2 & $1 \mathbf{a}\left(4-\mathrm{FC}_{6} \mathrm{H}_{5} / \mathrm{CH}_{3}\right)$ & $2 \mathbf{b}\left(\mathrm{C}_{6} \mathrm{H}_{5}\right)$ & $3 \mathbf{b}$ & 58 \\
\hline 3 & $\mathbf{1 b}\left(3-\mathrm{FC}_{6} \mathrm{H}_{5} / \mathrm{CH}_{3}\right)$ & $2 \mathbf{b}\left(\mathrm{C}_{6} \mathrm{H}_{5}\right)$ & $3 c$ & 62 \\
\hline 4 & $1 \mathrm{c}\left(3-\mathrm{ClC}_{6} \mathrm{H}_{5} / \mathrm{CH}_{3}\right)$ & $2 \mathbf{a}\left(4-\mathrm{ClC}_{6} \mathrm{H}_{5}\right)$ & $3 d$ & 63 \\
\hline 5 & $1 \mathrm{c}\left(3-\mathrm{ClC}_{6} \mathrm{H}_{5} / \mathrm{CH}_{3}\right)$ & $2 b\left(\mathrm{C}_{6} \mathrm{H}_{5}\right)$ & $3 e$ & 68 \\
\hline 6 & $1 d\left(4-\mathrm{BrC}_{6} \mathrm{H}_{5} / \mathrm{CH}_{3}\right)$ & $2 \mathbf{a}\left(4-\mathrm{ClC}_{6} \mathrm{H}_{5}\right)$ & $3 \mathbf{f}$ & 65 \\
\hline 7 & 1d $\left(4-\mathrm{BrC}_{6} \mathrm{H}_{5} / \mathrm{CH}_{3}\right)$ & $2 \mathbf{b}\left(\mathrm{C}_{6} \mathrm{H}_{5}\right)$ & $3 \mathbf{g}$ & 62 \\
\hline 8 & 1e $\left(3-\mathrm{BrC}_{6} \mathrm{H}_{5} / \mathrm{CH}_{3}\right)$ & $2 \mathbf{b}\left(\mathrm{C}_{6} \mathrm{H}_{5}\right)$ & $3 \mathbf{h}$ & 60 \\
\hline 9 & 1f $\left(\mathrm{C}_{6} \mathrm{H}_{5} / \mathrm{CH}_{3}\right)$ & $2 \mathbf{a}\left(4-\mathrm{ClC}_{6} \mathrm{H}_{5}\right)$ & $3 \mathbf{i}$ & 65 \\
\hline 10 & If $\left(\mathrm{C}_{6} \mathrm{H}_{5} / \mathrm{CH}_{3}\right)$ & $2 \mathbf{b}\left(\mathrm{C}_{6} \mathrm{H}_{5}\right)$ & $3 \mathbf{j}$ & 63 \\
\hline 11 & If $\left(\mathrm{C}_{6} \mathrm{H}_{5} / \mathrm{CH}_{3}\right)$ & $2 \mathrm{c}\left(4-\mathrm{OCH}_{3} \mathrm{C}_{6} \mathrm{H}_{5}\right)$ & $3 \mathbf{k}$ & 64 \\
\hline 12 & $\mathbf{1 g}\left(3-\mathrm{CH}_{3} \mathrm{C}_{6} \mathrm{H}_{5} / \mathrm{CH}_{3}\right)$ & $2 \mathbf{b}\left(\mathrm{C}_{6} \mathrm{H}_{5}\right)$ & 31 & 62 \\
\hline 13 & 1h (n-butyl/ $\left.\mathrm{CH}_{3}\right)$ & $2 \mathbf{a}\left(4-\mathrm{ClC}_{6} \mathrm{H}_{5}\right)$ & $3 \mathrm{~m}$ & 70 \\
\hline 14 & $\mathbf{1 i}\left(4-\mathrm{ClC}_{6} \mathrm{H}_{5} / \mathrm{H}\right)$ & $2 \mathbf{a}\left(4-\mathrm{ClC}_{6} \mathrm{H}_{5}\right)$ & $3 n$ & 71 \\
\hline 15 & $\mathbf{1 j}\left(3-\mathrm{ClC}_{6} \mathrm{H}_{5} / \mathrm{H}\right)$ & $2 \mathbf{a}\left(4-\mathrm{ClC}_{6} \mathrm{H}_{5}\right)$ & 30 & 72 \\
\hline 16 & 1k $\left(3-\mathrm{BrC}_{6} \mathrm{H}_{5} / \mathrm{H}\right)$ & $2 \mathrm{c}\left(4-\mathrm{OCH}_{3} \mathrm{C}_{6} \mathrm{H}_{5}\right)$ & $3 p$ & 68 \\
\hline 17 & $11\left(4-\mathrm{CH}_{3} \mathrm{C}_{6} \mathrm{H}_{5} / \mathrm{H}\right)$ & $2 \mathbf{a}\left(4-\mathrm{ClC}_{6} \mathrm{H}_{5}\right)$ & $3 q$ & 60 \\
\hline 18 & $\mathbf{1 a}\left(4-\mathrm{FC}_{6} \mathrm{H}_{5} / \mathrm{CH}_{3}\right)$ & 2d (n-propyl) & $3 \mathbf{r}$ & 62 \\
\hline
\end{tabular}

${ }^{a}$ The reaction was performed with $1(1.0 \mathrm{mmol}), 2(0.6 \mathrm{mmol})$, and $p$ TSA $(0.3 \mathrm{mmol})$ in EtOH $(15 \mathrm{~mL})$ at reflux for 3 hours. ${ }^{b}$ Isolated yields based on enaminones 1 . 
demonstrate that the process tolerates both 3-amino-5,5dimethylcyclohex-2-enones $\mathbf{1}$ and aromatic aldehydes $\mathbf{2}$ bearing either electron-donating or electron-withdrawing substituents on the aryl group. However, as both compounds $\mathbf{3}$ and $\mathbf{4}$ are generated from this reaction, the yields of $\mathbf{4}$ would be relatively low (yields $3: 4, c . a 1: 1$ ).

Next, the reaction was examined using $\mathrm{N}$-substituted 3aminocyclohex-2-enonephenyls. Unfortunately, the results did not yield much success. Most of the $\mathrm{N}$-substituted 3-aminocyclohex-2enonephenyls generated a low yield of $\mathbf{4}$ but a high yield of 3 (Scheme 2, $\mathbf{4 s - 4 u}$ ). It is worthwhile noting that compounds 4 are difficult to separate by conventional methods, like column chromatography and recrystallization. Hitherto, only three compounds were readily obtained by using $\mathrm{N}$-substituted 3-aminocyclohex-2enonephenyls.

Finally, aliphatic aldehydes butyraldehyde and cyclohexanecarboxaldehyde were also employed in this process, and both reactions proceeded well giving the corresponding 1,4-DHPs $\mathbf{4 v}$ and $\mathbf{4 w}$. And it was observed that the aliphatic aldehydes gave the major compounds 4 while products 3 were scarcely generated.

The chemical structures of 1,4-DHPs 3 and 4 were characterized by IR, ${ }^{1} \mathrm{H} \mathrm{NMR},{ }^{13} \mathrm{C}$ NMR, and HRMS, and unequivocally confirmed by X-ray diffraction analysis of a monocrystal of $\mathbf{3 n}$ (Fig. 1, CCDC 1819954).

The possible mechanism for the formation of products $3 / 4$ is shown in Scheme 3. Take aromatic aldehyde as the example. First, the benzaldehyde 2 accepts a proton to form 5. Enaminone $\mathbf{1}$ serves as a heteroene component to react with $\mathbf{5}$ accompanying the loss of one molecule of $\mathrm{H}_{2} \mathrm{O}$ to generate intermediate 6 via an aza-ene reaction. Following this, compound 6 undergoes imine-enamine tautomerization to produce 7 . Intermediate 7 accepts a proton to form $\mathbf{8}$, and the elimination of water from intermediate 7 gives iminium ion $\mathbf{9}$. Enaminone 1 then participates in a Michael addition with compound 9 to form intermediate 10. Two potential reactions then occur in intermediate 10. In pathway 1 , the $-\mathrm{NH}$ group

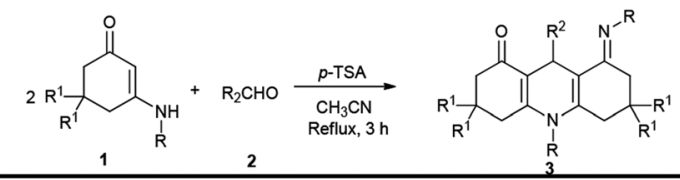

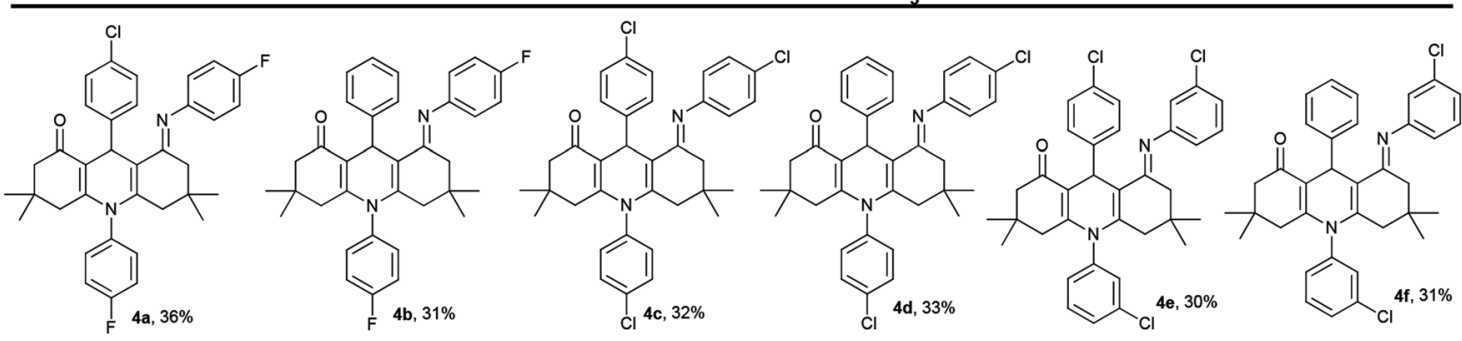

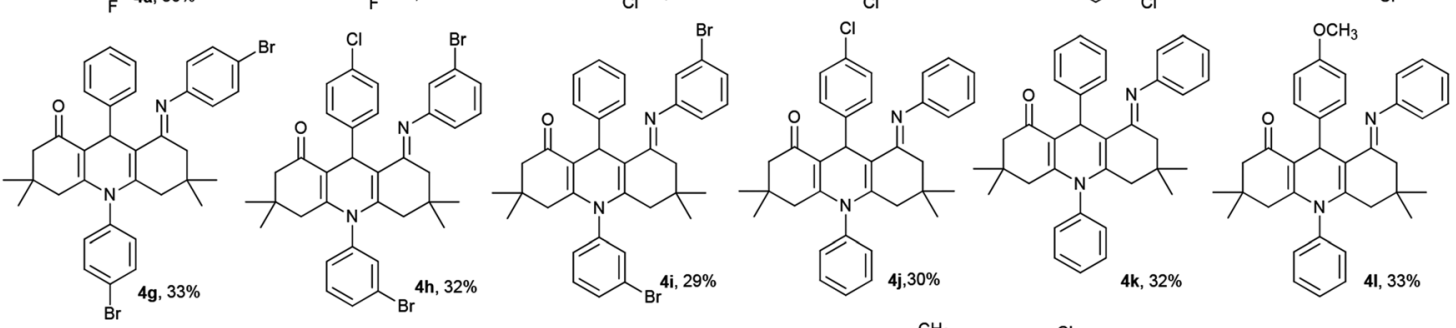

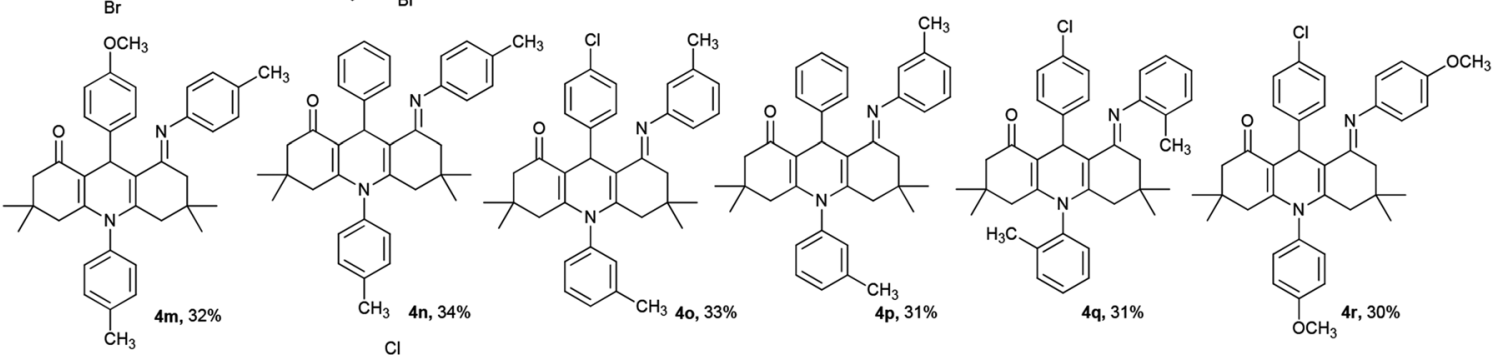<smiles>C[C@H](O)c1cccc(N2C3=C(C(=O)CCC3)C(c3ccc(Cl)cc3)C3=C2CCCC3=Nc2cccc(Cl)c2)c1</smiles><smiles>Cc1ccc(N=C2CCCC3=C2C(c2ccc(Cl)cc2)C2=C(CCCC2=O)N3c2ccc(C)cc2)cc1</smiles><smiles>O=C1CCCC2=C1C(c1ccc(Cl)cc1)C1C(=N2)CCCC1=Nc1ccccc1</smiles><smiles>CCCC1C(=O)CC(C)(C)Cc2c1c1c(n2-c2ccc(F)cc2)CC(C)(C)CC1=Nc1ccc(F)cc1</smiles><smiles>CC(C)c1ccc(N2C3=C(C(=O)CC(C)(C)C3)C(C3CCCCC3)C3=C2CC(C)(C)CC3=Nc2ccc(F)cc2)cc1</smiles>

Scheme 2 Substrate scope of the reaction for 1,4-DHPs 4 . The reaction was performed with 1 (1.0 mmol), $2(0.6 \mathrm{mmol})$, and $p$-TSA (0.3 mmol) in $\mathrm{CH}_{3} \mathrm{CN}(10 \mathrm{~mL})$ at reflux for $2 \mathrm{~h}$. The yields were isolated based on enaminones 1. 


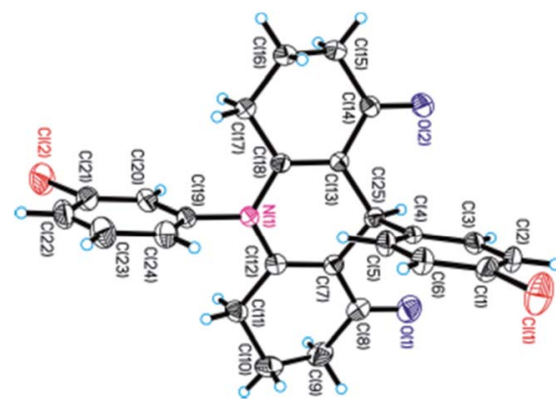

Fig. 1 X-ray crystal structures of $3 n$; ellipsoids are drawn at $30 \%$ probability level.
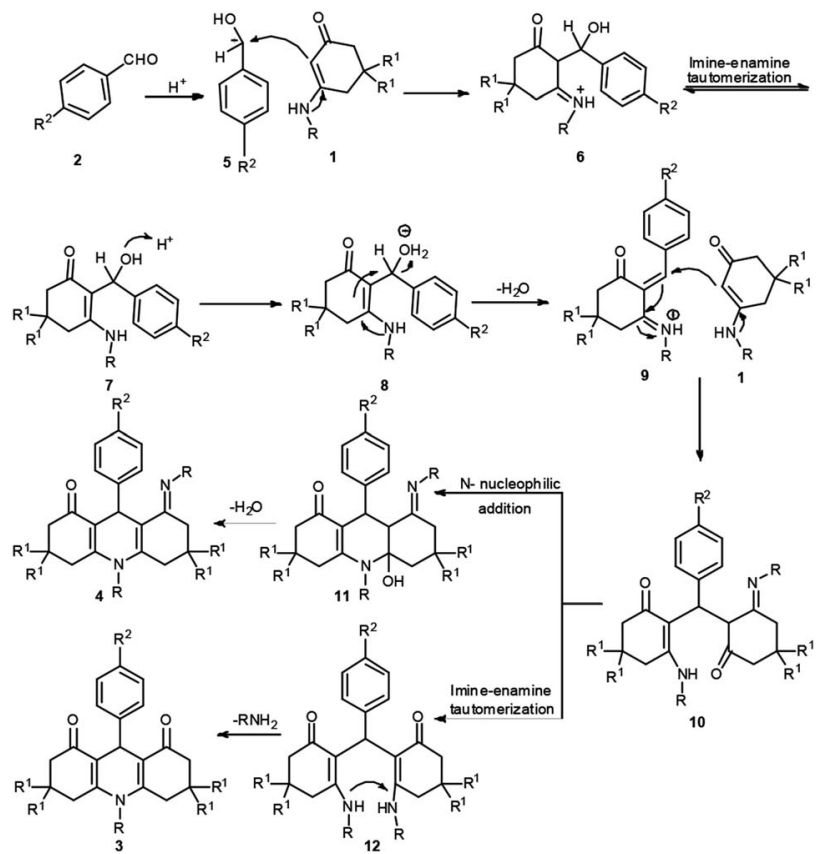

Scheme 3 Proposed mechanism for the construction of 1,4-DHPs 3 and 4 .

undergoes an intramolecular attack of the carbonyl group, resulting in a cyclization reaction that forces it to lose a molecule of water, affording 4. In pathway 2, intermediate 10 undergoes imine-enamine tautomerization to give intermediate 12 and elimination of aniline gives 1,4-DHPs 3.

\section{Conclusions}

In conclusion, we have successfully developed an operationally simple, one-pot three component reaction for the selective synthesis of two libraries of highly functionalized 1,4-DHPs derivatives by using of EtOH or $\mathrm{CH}_{3} \mathrm{CN}$ as media via a tandem cyclization of enaminones 1 with aldehydes 2 . The reaction displays many attractive features, including excellent regioselectivity, mild and environmentally friendly conditions, and molecular diversities. Moreover, these series of 1,4-DHPs may provide potential biological activities for medical treatment.
Further studies will be conducted in our laboratories in due course.

\section{Experimental}

\section{General}

All compounds were fully characterized by spectroscopic data. The NMR spectra were recorded on Bruker AVIII-400 ( ${ }^{1} \mathrm{H}: 400$ $\left.\mathrm{MHz},{ }^{13} \mathrm{C}: 100 \mathrm{MHz}\right)$ or Bruker AVIII-500 $\left({ }^{1} \mathrm{H}: 500 \mathrm{MHz},{ }^{13} \mathrm{C}: 125\right.$ $\mathrm{MHz})$ and chemical shifts $(\delta)$ are expressed in ppm, and $J$ values are given in $\mathrm{Hz}, \mathrm{CDCl}_{3}$ was used as solvent. IR spectra were recorded on a FT-IR Thermo Nicolet Avatar 360 using a $\mathrm{KBr}$ pellet. The reactions were monitored by thin-layer chromatography (TLC) using silica gel $\mathrm{GF}_{254}$. The melting points were determined on XT-4A melting point apparatus and are uncorrected. HRMS were performed on a Agilent LC/Msd TOF and monoisotopic mass instrument.

\section{General procedure for the synthesis of compounds 3}

A mixture of enaminones $1(1.0 \mathrm{mmol})$, aldehydes $2(0.6 \mathrm{mmol})$, p-TSA ( $0.3 \mathrm{mmol})$, and EtOH $(15 \mathrm{~mL})$ was stirred at reflux for $3 \mathrm{~h}$. After the desired product was formed as indicated by TLC, the reaction mixture was quenched with saturated $\mathrm{NH}_{4} \mathrm{Cl}$ solution (2 $\mathrm{mL})$ and extracted with ethyl acetate $(40 \mathrm{~mL})$. The organic phase was dried over $\mathrm{Mg}_{2} \mathrm{SO}_{4}$, and concentrated under vacuum. The residue was purified by flash chromatography (petroleum ether/ethyl acetate $=2: 3$ ) giving a white solid 3 .

9-(4-chlorophenyl)-10-(4-fluorophenyl)-3,3,6,6-tetramethyl3,4,6,7,9,10-hexahydroacridine-1,8(2H,5H)-dione (3a). White solid; mp 296-298 ${ }^{\circ} \mathrm{C}$; IR (KBr): 2959, 1649, 1618, 1500, 1371, $1226 \mathrm{~cm}^{-1} ;{ }^{1} \mathrm{H}$ NMR (400 MHz, $\left.\mathrm{CDCl}_{3}\right): \delta=7.35(\mathrm{~d}, J=8.4 \mathrm{~Hz}$, 2H, ArH), 7.20-7.29 (m, 6H, ArH), 5.24 (s, 1H, CH), 2.03-2.22 (m, 6H, $\left.\mathrm{CH}_{2}\right), 1.78-1.83\left(\mathrm{~m}, 2 \mathrm{H}, \mathrm{CH}_{2}\right), 0.96\left(\mathrm{~s}, 6 \mathrm{H}, 2 \times \mathrm{CH}_{3}\right), 0.81(\mathrm{~s}$, $\left.6 \mathrm{H}, 2 \times \mathrm{CH}_{3}\right) ;{ }^{13} \mathrm{C} \mathrm{NMR}\left(100 \mathrm{MHz}, \mathrm{CDCl}_{3}\right): \delta=195.7,163.6(\mathrm{~d}$, $\left.{ }^{1} J_{\mathrm{C}-\mathrm{F}}=249.8 \mathrm{~Hz}\right), 149.7,144.6,134.9,131.6,130.9,129.5,129.3$, 128.2, 117.3, 114.5, 50.1, 41.9, 32.4, 32.4, 29.7, 26.8; HRMS (ESITOF): $m / z$ calcd for $\mathrm{C}_{29} \mathrm{H}_{30} \mathrm{ClFNO}_{2}^{+}\left[(\mathrm{M}+\mathrm{H})^{+}\right]$, 478.1944; found, 478.1943.

10-(4-fluorophenyl)-3,3,6,6-tetramethyl-9-phenyl3,4,6,7,9,10hexahydroacridine-1,8(2H,5H)-dione (3b). White solid; mp 298$299{ }^{\circ} \mathrm{C}$; IR (KBr): 2959, 1641, 1510, 1402, 1220, $700 \mathrm{~cm}^{-1} ;{ }^{1} \mathrm{H}$ NMR (400 MHz, $\mathrm{CDCl}_{3}$ ): $\delta=7.43(\mathrm{~d}, J=8.0 \mathrm{~Hz}, 2 \mathrm{H}, \mathrm{ArH}), 7.24-$ 7.30 (m, 6H, ArH), 7.10-7.13 (m, 1H, ArH), 5.29 (s, 1H, CH), 2.21 $\left(\mathrm{AB}, J=16.0 \mathrm{~Hz}, 2 \mathrm{H}, \mathrm{CH}_{2}\right), 2.14\left(\mathrm{AB}, J=16.0 \mathrm{~Hz}, 2 \mathrm{H}, \mathrm{CH}_{2}\right), 2.08$ $\left(\mathrm{AB}, J=16.0 \mathrm{~Hz}, 2 \mathrm{H}, \mathrm{CH}_{2}\right), 1.83\left(\mathrm{AB}, J=16.0 \mathrm{~Hz}, 2 \mathrm{H}, \mathrm{CH}_{2}\right), 0.97$ $\left(\mathrm{s}, 6 \mathrm{H}, 2 \times \mathrm{CH}_{3}\right), 0.82\left(\mathrm{~s}, 6 \mathrm{H}, 3 \times \mathrm{CH}_{3}\right) ;{ }^{13} \mathrm{C} \mathrm{NMR}(100 \mathrm{MHz}$, $\left.\mathrm{CDCl}_{3}\right): \delta=195.7,162.5\left(\mathrm{~d},{ }^{1} J_{\mathrm{C}-\mathrm{F}}=249.0 \mathrm{~Hz}\right), 149.5,146.0$, 135.1, 135.0, 131.8, 128.1, 127.8, 126.0, 117.2, 114.8, 50.2, 41.9, 32.6, 32.4, 29.8, 26.8; HRMS (ESI-TOF): $\mathrm{m} / \mathrm{z}$ calcd for $\mathrm{C}_{29} \mathrm{H}_{31} \mathrm{FNO}_{2}^{+}\left[(\mathrm{M}+\mathrm{H})^{+}\right], 444.2333$; found, 444.2332.

\section{General procedure for the synthesis of compounds 4}

A mixture of enaminones 1 (1.0 $\mathrm{mmol})$, aldehydes $2(0.6 \mathrm{mmol})$, p-TSA $(0.3 \mathrm{mmol})$, and $\mathrm{CH}_{3} \mathrm{CN}(10 \mathrm{~mL})$ was stirred at reflux for $2 \mathrm{~h}$. After the desired product was formed as indicated by TLC, the reaction mixture was quenched with saturated $\mathrm{NH}_{4} \mathrm{Cl}$ 
solution $(2 \mathrm{~mL})$ and extracted with ethyl acetate $(40 \mathrm{~mL})$. The organic phase were dried over $\mathrm{Mg}_{2} \mathrm{SO}_{4}$, and concentrated under vacuum. The residue was purified by flash chromatography (petroleum ether/ethyl acetate $=1: 1$ ) giving a yellow solid 4.

$(E)-9-(4-c h l o r o p h e n y l)-10-(4-f l u o r o p h e n y l)-8-((4-f l u o-$ rophenyl)imino)-3,3,6,6-tetramethyl-3,4,5,6,7,8,9,10-octahydroacridin-1(2H)-one (4a). Yellow solid; mp 260-262 ${ }^{\circ} \mathrm{C}$; IR (KBr): 2959, 1649, 1500, 1371, 1226, $846 \mathrm{~cm}^{-1} ;{ }^{1} \mathrm{H}$ NMR (400 $\left.\mathrm{MHz}, \mathrm{CDCl}_{3}\right): \delta=7.41(\mathrm{t}, J=8.0 \mathrm{~Hz}, 2 \mathrm{H}, \mathrm{ArH}), 7.17-7.27(\mathrm{~m}, 6 \mathrm{H}$, ArH), 6.87-6.93 (m, 2H, ArH), 6.41-6.47 (m, 2H, ArH), 5.54 (s, $1 \mathrm{H}, \mathrm{CH}), 5.25(\mathrm{~d}, J=32.4 \mathrm{~Hz}, 1 \mathrm{H}, \mathrm{CH}), 1.92-2.24(\mathrm{~m}, 6 \mathrm{H}, 3 \times$ $\left.\mathrm{CH}_{2}\right), 1.67-1.83\left(\mathrm{~m}, 2 \mathrm{H}, \mathrm{CH}_{2}\right), 0.95\left(\mathrm{~s}, 3 \mathrm{H}, \mathrm{CH}_{3}\right), 0.85(\mathrm{~s}, 3 \mathrm{H}$, $\left.\mathrm{CH}_{3}\right), 0.83\left(\mathrm{~s}, 3 \mathrm{H}, \mathrm{CH}_{3}\right), 0.78\left(\mathrm{~s}, 3 \mathrm{H}, \mathrm{CH}_{3}\right) ;{ }^{13} \mathrm{C} \mathrm{NMR}(100 \mathrm{MHz}$, $\left.\mathrm{CDCl}_{3}\right): \delta=195.8,162.4\left(\mathrm{~d},{ }^{1} J_{\mathrm{C}-\mathrm{F}}=245.0 \mathrm{~Hz}\right), 158.8\left(\mathrm{~d},{ }^{1} J_{\mathrm{C}-\mathrm{F}}=\right.$ $238.5 \mathrm{~Hz}), 150.3,148.0,145.4,142.0,145.4,142.0,135.4,131.4$, 131.1, 129.6, 127.8, $120.7\left(\mathrm{~d},{ }^{3} \mathrm{~J}_{\mathrm{C}-\mathrm{F}}=7.5 \mathrm{~Hz}\right), 120.6\left(\mathrm{~d},{ }^{3} \mathrm{~J}_{\mathrm{C}-\mathrm{F}}=7.5\right.$ $\mathrm{Hz}), 117.0\left(\mathrm{~d},{ }^{2} J_{\mathrm{C}-\mathrm{F}}=21.5 \mathrm{~Hz}\right), 115.4\left(\mathrm{~d},{ }^{2} J_{\mathrm{C}-\mathrm{F}}=21.9 \mathrm{~Hz}\right), 115.1(\mathrm{~d}$, $\left.{ }^{2} J_{\mathrm{C}-\mathrm{F}}=21.9 \mathrm{~Hz}\right), 114.6,113.1,50.2,41.9,40.8,33.2,32.4,31.4$, 29.9, 29.6, 26.7; HRMS (ESI-TOF): $m / z$ calcd for $\mathrm{C}_{35} \mathrm{H}_{34} \mathrm{ClF}_{2} \mathrm{~N}_{2} \mathrm{O}^{+}$ $\left[(\mathrm{M}+\mathrm{H})^{+}\right], 571.2322$; found, 571.2323.

(E)-10-(4-fluorophenyl)-8-((4-fluorophenyl)imino)-3,3,6,6-tetramethyl-9-phenyl-3,4,5,6,7,8,9,10-octahydroacridin-1(2H)-one (4b). Yellow solid; mp $233-234{ }^{\circ} \mathrm{C}$; IR (KBr): 2957, 1644, 1579, 1367, 1267, $853 \mathrm{~cm}^{-1} ;{ }^{1} \mathrm{H}$ NMR (400 MHz, $\left.\mathrm{CDCl}_{3}\right): \delta=7.51(\mathrm{~d}, J$ $=8.0 \mathrm{~Hz}, 2 \mathrm{H}, \mathrm{ArH}), 7.24-7.28(\mathrm{~m}, 6 \mathrm{H}, \mathrm{ArH}), 7.15-7.11(\mathrm{~m}, 1 \mathrm{H}$, ArH), 6.91-6.95 (m, 2H, ArH), 6.48 (d, J=8.0 Hz, 2H, ArH), 5.63 (s, $1 \mathrm{H}, \mathrm{CH}), 2.01-2.27\left(\mathrm{~m}, 6 \mathrm{H}, 3 \times \mathrm{CH}_{2}\right), 1.81-1.87\left(\mathrm{~m}, 2 \mathrm{H}, \mathrm{CH}_{2}\right)$, $0.99\left(\mathrm{~s}, 3 \mathrm{H}, \mathrm{CH}_{3}\right), 0.88\left(\mathrm{~s}, 3 \mathrm{H}, \mathrm{CH}_{3}\right), 0.83\left(\mathrm{~s}, 3 \mathrm{H}, \mathrm{CH}_{3}\right), 0.74(\mathrm{~s}, 3 \mathrm{H}$, $\left.\mathrm{CH}_{3}\right) ;{ }^{13} \mathrm{C}$ NMR $\left(100 \mathrm{MHz}, \mathrm{CDCl}_{3}\right): \delta=195.9,162.3\left(\mathrm{~d},{ }^{1} J_{\mathrm{C}-\mathrm{F}}=\right.$ $249.0 \mathrm{~Hz}$ ), 158.8 (d, $\left.{ }^{1} J_{\mathrm{C}-\mathrm{F}}=238.0 \mathrm{~Hz}\right), 150.19,148.21,146.69$, 141.85, 135.57, 135.53, 131.59, 128.18, 127.69, 125.57, 120.72 (d, $\left.{ }^{3} J_{\mathrm{C}-\mathrm{F}}=8.0 \mathrm{~Hz}\right), 116.9\left(\mathrm{~d},{ }^{2} J_{\mathrm{C}-\mathrm{F}}=21.3 \mathrm{~Hz}\right), 115.3\left(\mathrm{~d},{ }^{2} J_{\mathrm{C}-\mathrm{F}}=22.0\right.$ $\mathrm{Hz}), 115.1\left(\mathrm{~d},{ }^{2} J_{\mathrm{C}-\mathrm{F}}=22.0 \mathrm{~Hz}\right), 114.9,113.4,50.3,41.9,41.8,40.8$, 33.5, 32.4, 31.5, 30.0, 29.6, 26.8, 26.69; HRMS (ESI-TOF): $\mathrm{m} / \mathrm{z}$ calcd for $\mathrm{C}_{35} \mathrm{H}_{35} \mathrm{~F}_{2} \mathrm{~N}_{2} \mathrm{O}^{+}\left[(\mathrm{M}+\mathrm{H})^{+}\right]$, 537.2712; found, 537.2713.

\section{Conflicts of interest}

There are no conflicts to declare.

\section{Acknowledgements}

This work was supported by the National Natural Science Foundation of China (No. 61361002, 21662046), Applied Basic Research Project of Yunnan (2017FD156), and Yunnan Province Department of Education Fund (2016ZZX217).

\section{Notes and references}

1 (a) J. D. Sunderhaus, C. Dockendorff and S. F. Martin, Org. Lett., 2007, 9, 4223; (b) F. Liéby-Muller, T. Constantieux and J. Rodriguez, J. Am. Chem. Soc., 2005, 127, 17176; (c) C. Lamberth and J. Dinges, in Bioactive Heterocyclic Compound Classes: Pharmaceuticals, ed. C. Lamberth and J. Dinges, Wiley-VCH, Weinheim, 2012, ch. 1, pp. 3-5; (d) C. Hulme and V. Gore, Curr. Med. Chem., 2003, 10, 51.

2 H. M. Refat and A. A. Fadda, Eur. J. Med. Chem., 2013, 70, 419.
3 (a) R. Surendra Kumar, A. Idhayadhulla, A. Jamal Abdul Nasser, S. Kavimani and S. Indumathy, Indian J. Pharm. Sci., 2010, 72, 719; (b) B. B. Subudhi, P. K. Panda, S. P. Swain and P. Sarangi, Acta Pol. Pharm., 2009, 66, 147.

4 (a) A. Hilgeroth, Mini-Rev. Med. Chem., 2002, 2, 235; (b) C. K. Chu, V. S. Bhadti, K. J. Doshi, J. T. Etse, J. M. Gallo, F. D. Boudinot and R. F. Schinazi, J. Med. Chem., 1990, 33, 2188; (c) A. Hilgeroth and H. Lilie, Eur. J. Med. Chem., 2003, 38, 495.

5 (a) H. A. Abbas, W. A. El Sayed and N. M. Fathy, Eur. J. Med. Chem., 2010, 45, 973; (b) S. R. Morshed, K. Hashimoto, Y. Murotani, M. Kawase, A. Shah, K. Satoh, H. Kikuchi, H. Nishikawa, J. Maki and H. Sakagami, Anticancer Res., 2005, 25, 2033; (c) J. Robert and C. Jarry, J. Med. Chem., 2003, 46, 4805.

6 I. O. Donkor, X. Zhou, J. Schmidt, K. C. Agrawal and V. Kishore, Bioorg. Med. Chem., 1998, 6, 563.

7 V. Klusa, Drugs Future, 1995, 20, 135.

8 A. H. Li, S. Moro, N. Forsyth, N. Melman, X.-D. Ji and K. A. Jacobson, J. Med. Chem., 1999, 42, 706.

9 A. Mai, S. Valente, S. Meade, V. Carafa, M. Tardugno, A. Nebbioso, A. Galmozzi, N. Mitro, E. D. Fabiani, L. Altucci and A. Kazantsev, J. Med. Chem., 2009, 52, 5496.

10 (a) J.-P. Wan and Y.-Y. Liu, RSC Adv., 2012, 2, 9763; (b) G. Li and J. C. Antilla, Org. Lett., 2009, 11, 1075; (c) D. Zhang, L.-Z. Wu, L. Zhou, X. Han, Q.-Z. Yang, L.-P. Zhang and C.-H. Tung, J. Am. Chem. Soc., 2004, 126, 3440; (d) P. R. Girling, A. S. Batsanov, H. C. Shen and A. Whiting, Chem. Commun., 2012, 4893; (e) Y. Nakaike, N. Nishiwaki, M. Ariga and Y. Tobe, J. Org. Chem., 2014, 79, 2163; (f) J.-P. , Wan, R.-H. Zhou, Y.-Y. Liu and M.-Z. Cai, RSC Adv., 2013, 3, 2477.

11 (a) V. Estevez, M. Villacampa and J. C. Menendez, Chem. Soc. Rev., 2010, 39, 4402; (b) E. Ruijter, R. Scheffelaar and R. V. A. Orru, Angew. Chem., Int. Ed., 2011, 50, 6234; (c) N. Isambert, M. d. M. S. Duque, J. C. Plaquevent, Y. Genisson, J. Rodriguez and T. Constantieux, Chem. Soc. Rev., 2011, 40, 1347; (d) J. Yu, F. Shi and L.-Z. Gong, Acc. Chem. Res., 2011, 44, 1156; (e) B. Ganem, Acc. Chem. Res., 2009, 42, 463; (f) A. Dömling, Chem. Rev., 2006, 106, 17; $(g)$ J.-P. Wan, L. Gan and Y.-Y. Liu, Org. Biomol. Chem., 2017, 15, 9031.

12 (a) B. Stanovnik and J. Svete, Chem. Rev., 2004, 104, 2433; (b) J.-P. Wan and Y. Gao, Chem. Rec., 2016, 16, 1164.

13 (a) B. Jiang, X. Wang, H.-W. Xu, M.-S. Tu, S.-J. Tu and G.-G. Li, Org. Lett., 2013, 15, 1540; (b) W.-J. Hao, J.-Q. Wang, X.-P. Xu, S.-L. Zhang, S.-Y. Wang and S.-J. Ji, J. Org. Chem., 2013, 78, 12362; (c) X.-B. Chen, X.-Q. Wang, Y. Hong and W. Liu, Asian J. Org. Chem., 2016, 5, 907; (d) H.-Y. Wang, L.-L. Li, W. Lin, P. Xu, Z.-B. Huang and D.-Q. Shi, Org. Lett., 2012, 14, 4598.

14 (a) H. Xu, B. Zhou, P. Zhou, J. Zhou, Y.-H. Shen, F.-C. Yu and L.-L. Li, Chem. Commun., 2016, 52, 8002; (b) X.-B. Chen, T.-B. Luo, G.-Z. Gou, J. Wang and W. Liu, Asian J. Org. Chem., 2015, 4, 921; (c) B. Jiang, M.-S. Yi, F. Shi, S.-J. Tu, S. Pindi, P. McDowell and G.-G. Li, Chem. Commun., 2012, 48, 808; (d) J.-D. Hu, C.-P. Cao, W. Lin, M.-H. Hu, 
Z.-B. Huang and D.-Q. Shi, J. Org. Chem., 2014, 79, 7935; (e) L. Wang, L.-X. Shi, L. Liu, Z.-X. Li, T. Xu, W.-J. Hao, G.-G. Li, S.-J. Tu and B. Jiang, J. Org. Chem., 2017, 82, 3605. 15 (a) J.-Y. Li, Q.-Y. Li, B. Jiang and S.-J. Tu, RSC Adv., 2013, 3, 5056; (b) B. Jiang, Y. Li, M.-S. Tu, S.-L. Wang, S.-J. Tu and G.-G. Li, J. Org. Chem., 2012, 77, 7497.

16 (a) M.-Y. Li, H.-W. Xu, W. Fan, Q. Ye, X. Wang, B. Jiang, S.-L. Wang and S.-J. Tu, Tetrahedron, 2014, 70, 1004; (b) F.-C. Yu, B. Zhou, H. Xu, Y.-M. Li, J. Lin, S.-J. Yan and Y.-H. Shen, Tetrahedron, 2015, 71, 1036; (c) X.-B. Chen, H.-R. Bai and C. Huang, Chin. J. Org. Chem., 2017, 31, 881; (d) X.-B. Chen, J.-W. Gong, X.-D. Zhang, X.-L. Liu, W. Liu and Y.-C. Wang, Tetrahedron, 2018, 74, 401.

17 (a) B. Jiang, X. Wang, M.-Y. Li, Q. Wu, Q. Ye, H.-W. Xu and S.-J. Tu, Org. Biomol. Chem., 2012, 10, 8533; (b) C.-P. Cao, W. Lin, M.-H. Hu, Z.-B. Huang and D.-Q. Shi, Chem. Commun., 2013, 49, 6983.

18 Z.-Y. Gu, J.-J. Gao, Y.-S. Wang and S.-J. Ji, Chem. Sci., 2016, 7, 4067.
19 (a) N. Ma, B. Jiang, G. Zhang, S.-J. Tu, W. Waver and G.-G. Li, Green Chem., 2010, 12, 1357; (b) S. Rana, M. Brownb and C. Mukhopadhyay, RSC Adv., 2013, 3, 3291; (c) F.-C. Yu, B. Zhou, H. Xu, K.-J. Chang and Y.-H. Shen, Tetrahedron Lett., 2015, 56, 837; (d) P. Zhou, B. Hu, J. Yang, L.-D. Li, K.-R. Rao, D.-F. Zhu and F.-C. Yu, Eur. J. Org. Chem., 2017, 29, 7256; (e) X.-J. Tu, W.-J. Hao, Q. Ye, S.-S. Wang, B. Jiang, G.-G. Li and S.-J. Tu, J. Org. Chem., 2014, 79, 11110; $(f)$ J.-P. Wan, Y.-F. Lin, Y.-F. Jin, M.-L. Xu and Y.-Y. Liu, Tetrahedron, 2014, 70, 7874; (g) S. Cao, S.-S. Zhong, C.-F. Hu, J.-P. Wan and C.-P. Wen, Chin. J. Chem., 2015, 33, 568.

20 X.-B. Chen, X.-Q. Wang, J.-N. Song, Q.-L. Yang, W. Liu and C. Huang, Org. Biomol. Chem., 2017, 15, 3611.

21 (a) J. A. Makawana, M. P. Patel and R. G. Patel, Chin. Chem. Lett., 2012, 23, 427; (b) C. Hundsdörfer, H. J. Hemmerling, C. Götz, F. Totzke, P. Bednarski, M. L. Borgne and J. Jose, Bioorg. Med. Chem., 2012, 20, 2282. 